\title{
SOCIAL (PRAGMATIC) COMMUNICATION DISORDERS AND AUTISM SPECTRUM DISORDER
}

Gillian Baird ${ }^{1}$ and Courtenay Frazier Norbury ${ }^{2}$

1 Newcomen Centre, Evelina, Guy's \& St Thomas NHS Trust, King's Health Partners

2 Division of Psychology \& Language Sciences, University College London

Summary

Changes have been made to the diagnostic criteria for autism spectrum disorder (ASD) in the recent revision of the Diagnostic and Statistical Manual of Mental Disorders (DSM-5) (1) and similar changes are likely in the World Health Organisation International Classification of Diseases (ICD-11) due in 2017. In light of these changes, a new clinical disorder, Social (pragmatic) Communication disorder (SPCD), was added to the Neurodevelopmental Disorders section of DSM-5. This article describes the key features of ASD, SPCD and the draft ICD11 approach to pragmatic language impairment, highlighting points of overlap between the disorders and criteria for differential diagnosis.

Introduction

Classification of diseases and disorders has been driven by the need for inter-rater reliability and for evidence based treatment. The ideal classification system has both validity and clinical utility. Validity concerns the extent to which diagnostic criteria correspond to a coherent pattern of symptoms that affect individuals in daily life. Clinical utility refers to the ability of a diagnosis to reduce adverse health outcomes by indicating a pathway to effective treatment and is a particular focus for the World Health Organisation (WHO). There are challenges to the classification of disorders which are clinically complex and for which there are limited biological-pathological correlates. For neurodevelopmental disorders such as autism spectrum disorder (ASD) there is likely a mix of genetic and epigenetic causation, with interaction of large numbers of common variants or penetrant single large mutations (SNPs or CNVs)(2). Other challenges are the dimensional nature of symptom profiles in many disorders with consequent imposition of categories on dimensions; the need to take into account developmental change over time in a life-span view of a disorder; and the recognition that co-morbidity is the norm, often blurring diagnostic boundaries. Issues of threshold are complex and challenging given that pathophysiology cannot be assessed directly and many 
symptoms of psychopathology are continuous with normal phenomena. Despite these challenges, diagnostic guidelines remain important clinical and research tools. The International Classification of Diseases (ICD,(3), produced by the World Health Organization in accordance with their mandate, and the Diagnostic and Statistical Manual of Mental Disorders (DSM,(1) are the two main diagnostic frameworks, providing definitions of clinical conditions, 'essential features' which differentiate a condition from other disorder and a threshold for diagnosis. In this paper we outline changes to the diagnostic criteria for ASD in both the DSM-5 and ICD-11 which prompted the introduction of a new diagnostic category, Social (Pragmatic) Communication Disorder (SPCD) in DSM-5 (see (4); (5). We describe SPCD and the related condition 'pragmatic language impairment' which is included as a language disorder in the draft ICD11; we consider the validity and clinical utility of these new diagnoses, review assessment in clinical settings and discuss issues concerning differential diagnosis. Finally, we briefly describe what is known about treatment for social/pragmatic difficulties.

A range of definitions are used both in clinical practice and scientific publications to describe pragmatic language and social communication (see Box 1 ) and at times the terms are used interchangeably. Some definitions of both include non-verbal communication (such as facial expression, gesture and tone of voice) and all modes or types of transmission between sender and receiver, including technology. Other definitions focus on social interaction and the social cognitive skills (i.e. theory of mind) that support successful social interaction. What is agreed is that pragmatic language skill is an essential component of social communication.

Box 1. Key language terminology.

1. 'Structural' language in this paper refers to semantics (meaning of words and texts) and the rules for producing and combining speech sounds (phonology) and combinations of words to form complex sentences (morphology and syntax e.g. walk + ed to form past tense). Language can be described as receptive or expressive.

2. Pragmatic language in this paper refers to the need to go beyond what is explicitly stated to reach the intended meaning or to resolve a structurally ambiguous message. Pragmatic skills typically require use of both the surrounding linguistic and social context. For example, the statement 'she is cold' can have very different meanings depending on the context. Jokes, figurative language, and inference are examples of pragmatic language skills.

3. Communication refers to the ability to receive, send and comprehend verbal, non-verbal and graphic symbol systems. Speech is only one form of communication.

4. Social communication in this paper uses Adams (6) definition: " the synergistic emergence of social interaction, social cognition, pragmatics (verbal and nonverbal), and receptive and 
expressive language processing" (p. 182). Non-verbal aspects of communication include tone of voice, facial expression and gesture). Social communication incorporates discourse skills such as turn taking, topic maintenance, adapting language to listener needs, providing maximally informative utterances without too much or too little detail, and strategies for repairing conversation.

\section{Changes to the diagnostic criteria for autism spectrum disorder}

In the most recent revision of DSM (DSM-5) and the forthcoming revision to ICD-11 (due 2017), diagnostic criteria for autism have changed in several significant ways (see Tables 1 and 2). Previous diagnostic classification systems (ICD-10:(3); DSM-IV-TR:(7) used the umbrella term pervasive developmental disorders and had a sub-classification structure that included childhood autism (ICD-10) or autistic disorder (DSM-IV), Asperger's syndrome/disorder, and pervasive developmental disorder - unspecified/not otherwise specified (PDD-NOS). In DSM-5 (and in draft proposals for ICD-11) these sub-classifications have been removed and are unified under the broader diagnostic category autism spectrum disorder (ASD). These changes arose from research demonstrating that the sub-classifications had little scientific justification and were used unreliably, even across expert teams (7). Autism spectrum disorder (ASD) is the new diagnostic term. The three domains of impairment are reduced to two core deficits: (A) social communication and social interaction (in which all criteria in this domain must be met) and (B) restricted and repetitive patterns of interests, behaviour or activities (in which two out of four criteria must be evident). Domain B now includes hyper-/hypo-reactivity to sensory stimuli and stereotyped language. Other critical features of the new DSM-5 definition are that symptoms may be present currently or by history, symptoms may not become apparent until social demands exceed capacity and symptoms cause significant functional impairment. Severity of symptoms in each domain is also described in DSM-5.

Table 1. Diagnostic Criteria for Autism Spectrum Disorder (DSM-5). Reprinted with permission from the Diagnostic and Statistical Manual of Mental Disorders, Fifth Edition, (Copyright 2013). American Psychiatric Association.

A. Persistent deficits in social communication and social interaction across multiple contexts, manifested by the following, currently or by history (examples are illustrative not exhaustive; see text):

1. Deficits in social-emotional reciprocity; ranging, for example, from abnormal social approach and failure of normal back-and-forth conversation; to reduced sharing of interests, emotions, or 
affect; to failure to initiate or respond to social interactions.

2. Deficits in nonverbal communicative behaviors used for social interaction, ranging, for example, from poorly integrated verbal and nonverbal communication; to abnormalities in eye contact and body language or deficits in understanding and use of gestures; to a total lack of facial expressions and nonverbal communication.

3. Deficits in developing, maintaining, and understanding relationships, ranging, for example, from difficulties adjusting behavior to suit various social contexts; to difficulties in sharing imaginative play or in making friends, to absence of interest in peers.

B. Restricted, repetitive patterns of behavior, interests, or activities, as manifested by at least two of the following, currently or by history (examples are illustrative, not exhaustive; see text):

1. Stereotyped or repetitive motor movements, use of objects, or speech (e.g., simple motor stereotypies, lining up toys or flipping objects, echolalia, idiosyncratic phrases).

2. Insistence on sameness, inflexible adherence to routines, or ritualized patterns of verbal or nonverbal behavior (e.g., extreme distress at small changes, difficulties with transitions, rigid thinking patterns, greeting rituals need to take same route or eat same food every day).

3. Highly restricted, fixated interests that are abnormal in intensity or focus (e.g., strong attachment to or preoccupation with unusual objects, excessively circumscribed or perseverative interests).

4. Hyper- or hypo-reactivity to sensory input or unusual interest in sensory aspects of the environment (e.g., apparent indifference to pain/temperature, adverse response to specific sounds or textures, excessive smelling or touching of objects, visual fascination with lights or movement).

C. Symptoms must be present in the early developmental period (but may not become fully manifest until social demands exceed limited capacities; or may be masked by learned strategies in later life).

D. Symptoms cause clinically significant impairment in social, occupational, or other important areas of current functioning.

E. These disturbances are not better explained by intellectual disability (intellectual developmental disorder) or global developmental delay. Intellectual disability and autism spectrum disorder frequently co-occur; to make comorbid diagnoses of autism spectrum disorder and intellectual disability, social communication should be below that expected for general developmental level.

Table 2. Definition and essential Features of Autism Spectrum Disorder (ICD-11 draft) 


\section{Printed with permission from the WHO.}

Definition: Autism Spectrum Disorder is characterized by persistent deficits in reciprocal social interaction and social communication, and by a range of restricted, repetitive, inflexible patterns of behaviour and interests together with sensory sensitivities. They may change in intensity, frequency and focus over the course of development. These deficits are usually a pervasive feature of the individual's functioning in all settings, although they may vary in degree according to the social, educational, or other context.

\section{Essential Features:}

Required features include persistent deficits in the capacity to initiate and to sustain reciprocal social interactions and social communication due to reduced interest or ability. There is limited understanding and use of language in context, and there are frequent inappropriate responses to other's verbal and nonverbal communication. There are deficits in shared interests, inappropriate responses to the emotional state of others and a lack of modulation of behaviour according to social context. There is poor integration of spoken language with nonverbal cues (such as eye contact, conventional gestures, facial expression, and body language). The ability to initiate and sustain social conversation is poor. The ability to form and to maintain friendships or other close relationships is impaired.

\section{Required features also include persistent restricted, repetitive, and inflexible patterns of} behaviour, interests, or activities

Distress over trivial changes to a familiar environment, or in response to unanticipated events, inflexible adherence to following particular routines, which may be geographic (e.g. following familiar routes) or which require precise timing (e.g. mealtimes, transport).

Excessive and persistent ritualistic patterns of behaviour, lining up or sorting objects in a particular way.

Repetitive and stereotyped motor movements, such as whole body movements (e.g. rocking), atypical gait (e.g., walking on tiptoes), unusual hand or finger movements and posturing, with repetitive and stereotyped language.

Excessive and persistent preoccupation with one or more special interests, with parts of objects, or other types of stimuli (e.g. film-clips or video-material including games), or an unusually strong attachment to particular objects (excluding typical comforters).

Excessive and persistent hypersensitivity or hyposensitivity to some sensory stimuli or unusual interests in a sensory stimulus, which may include actual or anticipated sounds, light, textures (especially clothing and food), odors and tastes, heat, cold, or pain.

Co-occurring conditions should be specified, e.g. co-occurring impairment of intellectual ability and functional language.

Individuals with Autism Spectrum Disorder may exhibit limitations in functional language. Functional 
language refers to the capacity of the individual to use language for instrumental purposes (e.g., to express personal needs and desires). Because of the social deficits that are intrinsic to Autism Spectrum Disorder, the assessment of functional language skills should place greater emphasis on instrumental usage rather than on social communication skills, because these will inevitably be impaired.

Social (Pragmatic) Communication Disorder.

A particular concern for the DSM-5 workgroup was the number of existing diagnoses using the 'not otherwise specified' PDD category from DSM-IV (referred to as 'atypical autism' in ICD-10). Individuals with subthreshold symptoms or two of three essential criteria, particularly social and communication deficits, could and did receive a diagnosis of PDD-NOS (8). There was concern that such individuals, with asocial communication impairment but without domain B symptoms, might be excluded from a DSM-5 ASD diagnosis and thus become ineligible for services. Both this group of individuals and individuals described in the existing literature as having "pragmatic language impairment" (9) were considered by the DSM-5 workgroup in the decision to include a new neurodevelopmental disorder, Social (Pragmatic) Communication Disorder (SPCD) (Table 3).

Table 3. Diagnostic Criteria for Social (Pragmatic) Communication Disorder (DSM-5, APA 2013) Reprinted with permission from the Diagnostic and Statistical Manual of Mental Disorders, Fifth Edition, (Copyright 2013). American Psychiatric Association.

A) Persistent difficulties in the social use of verbal and non-verbal communication as manifested by all of the following:-

1. Deficits in using communication for social purposes, such as greeting and sharing information, in a manner that is appropriate for the social context.

2. Impairment of the ability to change communication to match context or the needs of the listener, such as speaking differently in a classroom than on the playground, talking differently to a child than an adult and avoiding use of overly formal language.

3. Difficulties following rules for conversation and story-telling, such as taking turns in conversation, re-phrasing when misunderstood and knowing how to use verbal and non-verbal signals to regulate interaction.

4. Difficulties in understanding what is not explicitly stated, (for example making inferences). A non-literal or ambiguous means of language, for example, (idioms, humour, metaphors, multiple meanings which depend upon the context for the interpretation). 
B) The deficits result in functional limitations in effective communication, social participation, social relationships, academic achievement or occupational performance, individually or in combination.

C) The onset of the symptoms is in the early developmental period, but the deficits may not become fully manifest until social communication demands exceed limited capacity.

D) The symptoms are not attributable to another medical or neurological condition, or to low abilities in the domains of word structure and grammar and are not better explained by an autism spectrum disorder, intellectual disability (intellectual developmental disorder), global developmental delay or another mental disorder.

\section{How is SPCD differentiated from ASD?}

Happe and Ronald (10) discuss the degree to which the social interaction, communication, flexible imagination and restricted and repetitive behaviors and interests (RRBIs) are 'fractionable' within ASD. They cite evidence from twin studies that the different symptoms of ASD may be influenced by different genetic risk factors, cognitive components and neural patterns. Thus communication, social interaction and RRBIs may theoretically be separable and differently combined in ASD, reflecting the common clinical experience of some features being more marked than others in individuals, although patterns of co-occurrence may change over time. It is not yet clear how the criteria for SPCD differs from domain A of ASD. Criteria for SPCD explicitly include deficits in both verbal and non-verbal communication for social purpose. Both are also essential criteria for ASD as, by definition, individuals with ASD have a social communication disorder that disrupts conversational discourse. Deficits in pragmatic language are essential in SPCD, for instance making inferences and comprehending ambiguous language such as jokes and metaphors, although it is not clear how many will fulfil all four domain A criteria and there is evidence that these domains may dissociate (see (4)). While many individuals with ASD will have obvious pragmatic language deficits, some individuals with ASD who score within the normal range on measures of structural language may succeed in tasks purporting to test pragmatic language skill (for example, in resolving ambiguous phrases), despite pronounced challenges in maintaining social discourse (cf. (4). Impairment in social relationships is a core deficit in ASD. In contrast, individuals with SPCD are thought to have a typical social drive to communicate, though this may be challenging to establish.

Aspects of reciprocity and relationships such as 'sharing interests, emotions and affect', 'modifying behaviour (rather than language) to suit the social context' and imaginative ability may 
be differentiating features. However, in practice, the differential diagnosis of SPCD from ASD largely rests on the absence of rigid, restricted and repetitive interests, behaviours, and activities in both the history and current assessment of the individual. In ascertaining such symptoms, it is important to remember that individuals with ASD may display restricted repetitive patterns of behaviour interests and activities more obviously during the early developmental period and so a comprehensive history should be obtained. Other changes in the criteria for ASD in domain B of DSM-5, which now include stereotyped language, hypo and hyper sensory sensitivities, and rigidity of thinking (insistence on sameness), may also impact on diagnosis. This may change inclusion/exclusion for those individuals once thought to have a 'pragmatic language impairment' and many may meet new diagnostic criteria for ASD (4).

\section{Pragmatic language impairment}

The ICD-11 approach to including individuals with 'pragmatic language impairment' (9) has been to use a descriptive qualifier within Language Disorders (see Table 4 for draft ICD-11 guidelines). Those meeting criteria for Language Disorder represent a heterogeneous group of individuals with varying patterns of language strength and deficit. ICD-11 allows the clinician to characterise the main areas of language impairment using one of the following qualifiers:

1. Developmental Language Disorder, with impairment of receptive and expressive language

2. Developmental Language Disorder, with impairment of mainly expressive language

3. Developmental Language Disorder, with impairment of pragmatic language

\section{Table 4. DRAFT Clinical Description and Diagnostic Guidelines for Developmental Language Disorder (ICD-11). Printed with permission from WHO:}

\section{Definition:}

Developmental language disorder is characterized by persistent difficulties in the acquisition, understanding, production or use of language (spoken or signed), that arise during the developmental period, typically during early childhood, and cause significant limitations in the individual's ability to communicate. The individual's ability to understand or express language is markedly below what would be expected given the individual's age and level of intellectual functioning. The language deficits are not explained by another neurodevelopmental disorder or a sensory impairment or neurological condition, including the effects of brain injury or infection.

Inclusion Terms: Specific language impairment (SLI), language learning impairment, language development disorder 


\section{Exclusion Terms: Diseases of the Nervous System, Selective Mutism}

\section{Essential Features:}

- Persistent deficits in the acquisition, understanding, production or use of language (spoken or signed). Any of the components of language may be differentially impaired, with relative weaknesses in some aspects of language and relative strengths in others. These include overall receptive language (the ability to understand spoken or signed language) and overall expressive language (the ability to produce and use spoken or signed language), as well as a variety of more specific language abilities, including:

0 the ability to understand and use the meaning of words and sentences (semantics);

- the ability to apply language rules, for example regarding verb conjugation or word endings and how words are combined to form sentences (syntax or grammar);

○ the ability to decompose words into constituent sounds and mentally manipulate those sounds (phonological awareness);

- the ability to tell a story or have a conversation (narrative or conversational discourse);

○ the ability to understand and use language in social contexts, for example making inferences, understanding verbal humour and resolving ambiguous meaning (pragmatics)

- Language abilities are markedly below what would be expected on the basis of age and level of intellectual functioning.

- Onset of language difficulties occurs during the developmental period, typically during early childhood.

- The language deficits cause significant limitations in the ability to communicate.

- The language deficits are not explained by another neurodevelopmental disorder, a sensory impairment, or a neurological condition, including the effects of brain injury or infection (e.g., due to trauma, stroke, epilepsy, or meningitis).

Many children with structural language deficits also have difficulties with pragmatic language skills, because of the need to use linguistic context to access meaning (11). Nevertheless, DSM-5 states that symptoms of SPCD are 'not attributable to low abilities in the domains of word structure and grammar' and this would imply that children who exhibit pragmatic impairments and co-occurring 
structural language impairments would be excluded from a diagnosis of SPCD. The pragmatic language qualifier in ICD-11 is applied when the language disorder is characterized by persistent and substantial difficulties with pragmatic language skills that are not explained 'on the basis of other components of receptive and expressive language'. The intention was not necessarily to imply causality, but rather to identify, by clinical judgement, a co-occurring problem of a greater impairment in pragmatic skills than expected given the child's overall level of structural language ability. Cluster analyses of children who have predominantly structural language impairment have demonstrated that many clinically referred cases have far greater and more persistent impairments in pragmatic language skills (12). However, it can be difficult to benchmark the level of expected pragmatic ability for a given level of structural language skill, and there is an urgent need to examine reliability of diagnosis across practitioners.

A Comparison of Criteria for SPCD and pragmatic language impairment is summarised inTable 5.

\begin{tabular}{|l|l|l|}
\hline & $\begin{array}{l}\text { Social Pragmatic } \\
\text { Communication Disorder } \\
\text { (DSM-5) }\end{array}$ & $\begin{array}{l}\text { Developmental Language } \\
\text { Disorder with Pragmatic } \\
\text { Language Impairment (draft } \\
\text { for ICD-11) }\end{array}$ \\
\hline Core criteria & & \\
\hline $\begin{array}{l}\text { Deficits in narrative or } \\
\text { conversational discourse }\end{array}$ & Yes & Yes \\
\hline $\begin{array}{l}\text { Deficits in pragmatic } \\
\text { language, or going beyond } \\
\text { what is explicitly stated, i.e. } \\
\text { inference, figurative } \\
\text { language, ambiguity }\end{array}$ & Yes & Yes \\
\hline $\begin{array}{l}\text { Impaired ability to adjust } \\
\text { communication to meet } \\
\text { listener need }\end{array}$ & Yes & Not explicitly required, \\
\hline $\begin{array}{l}\text { Deficits in using language } \\
\text { for social purposes, i.e. } \\
\text { sharing information }\end{array}$ & Yes & Not required \\
\hline $\begin{array}{l}\text { Deficit in matching } \\
\text { communication to social } \\
\text { context }\end{array}$ & Yes & Not required \\
\hline Additional criteria & \multicolumn{2}{|l|}{} \\
\hline $\begin{array}{l}\text { Intellectual disability } \\
\text { Symptoms not better } \\
\text { explained by intellectual } \\
\text { ability }\end{array}$ & $\begin{array}{l}\text { Pragmatic deficit markedly } \\
\text { below level of intellectual } \\
\text { functioning }\end{array}$ \\
\hline $\begin{array}{l}\text { Structural language } \\
\text { impairment, i.e. impaired } \\
\text { phonology, vocabulary } \\
\text { and/or grammar }\end{array}$ & $\begin{array}{l}\text { to low ability in structural } \\
\text { language }\end{array}$ & $\begin{array}{l}\text { Pragmatic deficit greater } \\
\text { than expected for level of } \\
\text { structural language }\end{array}$ \\
\hline $\begin{array}{l}\text { Deficits in non-verbal } \\
\text { communication, i.e. eye } \\
\text { gaze, facial expression, }\end{array}$ & Essential & Not required, \\
\hline
\end{tabular}




\begin{tabular}{|l|l|l|}
\hline gesture & Required in Criterion B, \\
\hline $\begin{array}{l}\text { Impaired social } \\
\text { understanding and social } \\
\text { relationships }\end{array}$ & $\begin{array}{l}\text { arise as consequence of } \\
\text { SPCD }\end{array}$ & Not required \\
\hline Impaired communication & Yes & Yes \\
\hline $\begin{array}{l}\text { Restricted repetitive rigid } \\
\text { repertoire of activities, } \\
\text { interests and behaviours as } \\
\text { in Domain B for ASD }\end{array}$ & No & No \\
\hline
\end{tabular}

Both DSM-5 and ICD-11 criteria specify that for diagnosis, social communication and/or pragmatic language abilities are markedly below the expected level given the individual's age and level of intellectual functioning. Making a clinical judgement of deficit taking into account developmental age or intellectual/cognitive ability is included in all neurodevelopmental disorders although the implied causal relationship or limit on potential for change is controversial. This is a lively topic of debate as such exclusion criteria may limit access to treatment and the diagnostic boundaries around intellectual impairment remain arbitrary (13).

Functional impairment refers to the extent to which a diagnosis results in limitations in daily life, including social experiences and educational opportunities. There are no existing research studies that have documented the functional impairment associated with SPCD (14). However, a large body of research has investigated the correlates of pragmatic language impairment. These studies have consistently demonstrated that children with pragmatic deficits are at increased risk for developing behaviour problems (15)(14). Similarly, assessment of children referred to child and adolescent mental health services has revealed increased rates of social communication and pragmatic language deficit (16)(17)(18). Children with pragmatic language deficits may also have academic challenges, particularly with regard to reading comprehension, where the ability to make inferences and resolve ambiguities is critical to understanding text (19). Finally, pragmatic language deficits are persistent and are associated with adverse outcomes in adults (20).

\section{Age of recognition of SPCD.}

The recognition of SPCD, like ASD, is in the early developmental period, but the deficits may not become fully manifest until social communication demands exceed limited capacity and it is expected that the diagnosis would seldom be made until the age of about four or five when deficits in pragmatic language that are out of line with structural language abilities may be easier to identify. In other words, younger children may not have sufficient structural language skills to make inferences or tell coherent narratives, rendering judgement of pragmatic language unreliable. 
However children with any delay in language that is of concern to their parents or school teachers or which is having some impact on their understanding, communication and/or social interaction should be referred for assessment.

Assessment of social communication and pragmatic language skill.

Reliable assessment of social communication is challenging (21) both because social communication requires exchange between two individuals and there are few suitable assessment tools with adequate psychometric properties and relevant developmental norms. It is therefore difficult to measure skills in a standardised way and there is often reliance on parent and/or teacher report of communication in everyday contexts (22). The Children's Communication Checklist-2 (CCC-2, (22) is perhaps the most widely used in both clinical practice and research. The UK version of the CCC- 2 is comprised of 70 items over 10 sub-scales; eight sub-scales tap structural and pragmatic language and two scales measure restricted interests and deficits in social reciprocity that are more typical of ASD. Respondents are asked to rate the frequency of communication behaviours on a four-point scale. Normative data are available on over 500 UK children and more than 900 US children aged 4 to 17 years and it has been translated into more than 30 different languages.

From these ratings, the Social Interaction Deviance Composite (SIDC) is calculated, which identifies pragmatic abilities that are disproportionately impaired relative to structural language competencies. Thus, a positive score indicates relatively mild pragmatic difficulties in conjunction with more severe deficits in structural language; such a score would be more indicative of a specific language impairment. Scores around zero are seen in children with both severe pragmatic and structural language deficits, as is the case for many children with ASD. Negative scores would be more consistent with a profile in which scores on structural language tests were within normal limits, but the child experienced pronounced social communication deficits. In a validation study, scores on the SIDC were continuously distributed, with no clear categorical boundaries between specific language impairment, SPCD or ASD (11). Thus, the CCC-2 should be used to signpost aspects of communication for further assessment and is not intended as a diagnostic tool. Structured observation of conversation is also advocated, as in the Autism Diagnostic Observation Schedule-2 (23). The ADOS-2 includes ratings of social interaction, including eye gaze and response to bids for attention, as well as a direct measure of conversational exchange. However, the ADOS-2 covers a wide age range and there are limited normative data on which to base judgements of conversational adequacy. It is also very likely that conversational skill will depend in part on the structural language competencies of the child. 
Only one study has specifically investigated SPCD (14). The definition of pragmatics employed by Miller et al. included aspects of social knowledge and social exchange, features such as the child's ability to react to the attention of others, to talk and ask about other's behaviour and mental state, to tease and take the listener's knowledge into account (14). The Language Use Inventory (LUI; (24) was used to assess early pragmatic skills in a group of children aged 36 months, enrolled because they were at high risk of ASD. Children identified with possible SPCD had a higher rate of 'structural language impairment' although the majority, over $80 \%$ did not. In addition, most had elevated ADOS scores, although below diagnostic cut-off. The most frequently rated clinical outcome measure was 'broader autism phenotype', i.e. below-threshold ASD symptoms.

Unfortunately, restricted and repetitive interests and behaviours were not explicitly reported in this study. The authors also note that while children with pragmatic deficits had elevated symptoms of social, behavioural and language difficulties, they were not in the clinical range. This raises questions about the extent to which social communication and/or pragmatic language impairment gives rise to functional impairments in the absence of other developmental concerns.

Making a diagnosis of a disorder, ASD or SPCD or language disorder, using either DSM-5 or ICD 11 remains a clinical judgement best carried out by a multi-disciplinary team, which should ideally include a paediatrician, speech and language therapist and psychologist, using the available tools, however inadequate. Case history, questionnaires such as CCC-2, direct assessment of structural language using standardised tests and social communication using the ADOS are currently the best available methods.

Diagnosis ideally signposts treatment pathways. There is a dearth of intervention trials to inform treatment decisions in social communication/pragmatic language disorder (25). We know very little about the nature and efficacy of interventions for children with SPCD; whether treating social communication requires a different approach depending on whether children meet strict criteria for SPCD, or if the pragmatic deficits occur in conjunction with structural language impairment and/ or ASD. The only randomised controlled trial of intervention targeting social communication disorder did not show significant change on the primary outcome measure (25). However, the outcome measure did not specifically assess social communication, and the intervention groups included a heterogeneous cohort with and without co-morbid language disorder and/or ASD. Clearly improving assessment protocols and increasing robust trials of intervention for social communication and pragmatic language is a priority for future research.

\section{Conclusion.}


The intention is for DSM-5 and ICD-11 to be as similar as possible, to ensure similar standards of diagnosis across geographical boundaries. While this has largely been achieved for ASD, SPCD, a new diagnosis in DSM-5 whose validity and clinical utility remain to be established, has not currently been included in ICD-11. Instead pragmatic language disorder is a qualifier under the umbrella of Developmental Language Disorder. The diagnostic criteria of SPCD overlap considerably with the social communication domain of ASD. Children with SPCD are distinguished from those with ASD principally on the basis of lifetime absence of restricted and repetitive interests and behaviours although there may be other subtleties of language, communication, imagination and social behaviour as yet unclear. It is currently unknown how many individuals have pragmatic language impairment in the context of good grammar, vocabulary and word knowledge, without any current or previous history of rigid, repetitive and restrictive interests and behaviours, and in the absence of global cognitive delays. Norbury (4) has conceptualised SPCD as a dimensional symptom profile that may be evident across a range of developmental disorders, rather than a discrete diagnostic category. Nevertheless, increased focus on social communication and pragmatic language skills should prompt development of intervention approaches and clinically relevant outcome measures.

Acknowledgments: the criteria for ASD and SPCD from DSM-5 have been reprinted by kind permission of the APA. The WHO has also given permission for the inclusion of the draft ICD-11 criteria for ASD and Language Disorder. The proposed clinical descriptions for ICD-11 are currently undergoing field trials.

Declaration of interest: GB was a member of the DSM-5 working party and is a member of the ICD11 working party.

References

1. American Psychiatric Association. Diagnostic and Statistical Manual of Mental Disorders (5th ed.). Washington, DC: Author; 2013.

2. Kiser DP, Rivero O, Lesch K-P. Annual Research Review: The (epi)genetics of neurodevelopmental disorders in the era of whole-genome sequencing - unveiling the dark matter. J Child Psychol Psychiatry. 2015 Mar;56(3):278-95.

3. World Health Organisation. The ICD-10 classification of mental and behavioural disorders: Clinical descriptions and diagnostic guidelines. Geneva: World Health Organisation; 1992.

4. Norbury CF. Practitioner Review: Social (pragmatic) communication disorder conceptualization, evidence and clinical implications. J Child Psychol Psychiatry. 2014 Mar;55(3):204-16. 
5. Swineford LB, Thurm A, Baird G, Wetherby AM, Swedo S. Social (pragmatic) communication disorder: a research review of this new DSM-5 diagnostic category. $J$ Neurodev Disord. 2014;6(1):41.

6. Adams C. Social Communication Intervention for School-Age Children: Rationale and Description. Semin Speech Lang. 2005 Aug;26(03):181-8.

7. Lord C. A Multisite Study of the Clinical Diagnosis of Different Autism Spectrum Disorders. Arch Gen Psychiatry. 2012 Mar 1;69(3):306.

8. Skuse DH. DSM-5's Conceptualization of Autistic Disorders. J Am Acad Child Adolesc Psychiatry. 2012 Apr;51(4):344-6.

9. Bishop DVM, Norbury CF. Exploring the borderlands of autistic disorder and specific language impairment: a study using standardised diagnostic instruments. J Child Psychol Psychiatry. 2002 Oct;43(7):917-29.

10. Happé F, Ronald A. The "Fractionable Autism Triad": A Review of Evidence from Behavioural, Genetic, Cognitive and Neural Research. Neuropsychol Rev. 2008 Dec;18(4):287-304.

11. Norbury CF, Nash M, Baird G, Bishop DVM. Using a parental checklist to identify diagnostic groups in children with communication impairment: a validation of the Children's Communication Checklist-2. Int J Lang Commun Disord. 2004 Jan;39(3):345-64.

12. Conti-Ramsden G, Crutchley A, Botting N. The extent to which psychometric tests differentiate subgroups of children with SLI. J Speech Lang Hear Res JSLHR. 1997 Aug;40(4):765-77.

13. Reilly S, Bishop DVM, Tomblin B. Terminological debate over language impairment in children: forward movement and sticking points: Terminology for children with language problems. Int J Lang Commun Disord. 2014 Jul;49(4):452-62.

14. Miller M, Young GS, Hutman T, Johnson S, Schwichtenberg AJ, Ozonoff S. Early pragmatic language difficulties in siblings of children with autism: implications for DSM5 social communication disorder? J Child Psychol Psychiatry. 2015 Jul;56(7):774-81.

15. Mok PLH, Pickles A, Durkin K, Conti-Ramsden G. Longitudinal trajectories of peer relations in children with specific language impairment. J Child Psychol Psychiatry. 2014 May;55(5):516-27.

16. Gremillion ML, Martel MM. Merely Misunderstood? Receptive, Expressive, and Pragmatic Language in Young Children With Disruptive Behavior Disorders. J Clin Child Adolesc Psychol. 2014 Sep;43(5):765-76.

17. Im-Bolter N, Cohen NJ, Farnia F. I thought we were good: social cognition, figurative language, and adolescent psychopathology. J Child Psychol Psychiatry. 2013 Jul;54(7):724-32.

18. Im-Bolter N, Cohen NJ. Language Impairment and Psychiatric Comorbidities. Pediatr Clin North Am. 2007 Jun;54(3):525-42. 
19. Freed J, Adams C, Lockton E. Predictors of reading comprehension ability in primary school-aged children who have pragmatic language impairment. Res Dev Disabil. 2015 Jun;41-42:13-21.

20. Whitehouse AJO, Watt HJ, Line EA, Bishop DVM. Adult psychosocial outcomes of children with specific language impairment, pragmatic language impairment and autism. Int J Lang Commun Disord. 2009 Jan 1;44(4):511-28.

21. Adams C. Practitioner review: the assessment of language pragmatics. J Child Psychol Psychiatry. 2002 Nov;43(8):973-87.

22. Bishop DVM. Children's Communication Checklist-2. London: Pearson; 2003.

23. Lord C, Luyster RJ, Gotham K, Guthrie W. Autism diagnostic observation schedule, second edition (ADOS-2). 2nd ed. Torrance, CA: Western Psychological Services; 2012.

24. O'Neill DK. The language use inventory for young children: a parent-report measure of pragmatic language development for 18- to 47-month-old children. J Speech Lang Hear Res JSLHR. 2007 Feb;50(1):214-28.

25. Adams C, Lockton E, Freed J, Gaile J, Earl G, McBean K, et al. The Social Communication Intervention Project: a randomized controlled trial of the effectiveness of speech and language therapy for school-age children who have pragmatic and social communication problems with or without autism spectrum disorder. Int J Lang Commun Disord R Coll Speech Lang Ther. 2012 Jun;47(3):233-44. 